\title{
Effectiveness of the toxin-degrading strain screening strategy: Deficiency and improvement in screening the gossypol-degrading microorganism Aspergillus niger
}

Chen Zhang

Anhui Agricultural University

Gangqin Shu

Anhui Agricultural University

Hui Wang

Anhui Agricultural University

Daojie Li

Anhui Agricultural University

Yuan Xü

Anhui Agricultural University

Yunhua Zhang

Anhui Agricultural University

Maoji Cheng Cheng

Anhui Agricultural University

Lijuan Chen ( $\nabla$ zhangchenlijuan@163.com )

Anhui Agricultural University

\section{Research Article}

Keywords: Gossypol, Degrade, Aspergillus niger, Agarase, Screening strategy

Posted Date: September 3rd, 2021

DOI: https://doi.org/10.21203/rs.3.rs-152600/v2

License: (9) This work is licensed under a Creative Commons Attribution 4.0 International License.

Read Full License 


\section{Effectiveness of the toxin-degrading strain screening}

2 strategy: Deficiency and improvement in screening the

4 Chen Zhang ${ }^{1}$, Gangqin Shu ${ }^{1}$, Hui Wang ${ }^{1}$, Daojie Li $^{1}$, Yuan Xï ${ }^{1}$ Yunhua $5 \quad$ Zhang $^{2}$, Maoji Cheng ${ }^{1,3}$, Lijuan Chen $^{1 *}$ gossypol-degrading microorganism Aspergillus niger

${ }^{1}$ The Biological Feedstuff Lab, College of Animal science and Technology, Anhui Agricultural University, Hefei, Anhui 230036, P.R. China

${ }^{2}$ Anhui Province Key Lab of Farmland Ecological Conservation and Pollution Prevention, College of Resources and environment, Anhui Agricultural University, Hefei, Anhui 230036, P.R. China

${ }^{3}$ Fisugarpeptide Blology Engineering CO., LTD, Luan, Anhui 237000, P.R. China

* Correspondence: zhangchenlijuan@163.com; Tel.: +86-0551-65786328

\section{ABSTRACT}

The effectiveness of the classic screening strategy was verified, by duplicating and verifying the degradation of gossypol by the Aspergillus niger. It can reduce the free gossypol content through biosorption but has no effect on the total gossypol content and cannot effectively degrade gossypol. And the most interesting thing we found the strain can secrete agarase, utilise agar as carbon source. In this case, that will mislead researchers and lead them to make wrong judgments. That turns out the usual methods of previous screening strategies are not rigorous enough, the classic screening method has defect in screening toxin-degrading strain, so agar control group should be added. In this study, some suggestions are put forward to 
optimise the same type of experiments and broaden the idea of detoxification by microorganisms and provide reference for screening effective toxin-degrading microorganisms.

Keywords: Gossypol, Degrade, Aspergillus niger, Agarase, Screening strategy

\section{Introduction}

Toxic substances in soil, water, food and feed can be degraded by microorganisms (Ghazali et al., 2004; Hazrat et al., 2010; Ji et al., 2010;). Compared with physical and chemic methods, the biodegradation technology has the advantages of safety, efficiency, moderate, environmental friendliness, absence of secondary pollution and low cost and is the most promising treatment method that has been paid attention by researchers. The classic screening method (Fig.1 A) is that (Lewis et al., 2020): (1) Samples are usually collected from a variety of specific areas where degrading microorganisms may exist. (2) A certain toxic substance is used as the sole carbon (or nitrogen) and energy sources, and microorganisms are inoculated in an agar solid medium. (3)The degradation effect is judged using the diameter of the hydrolysis circle or the growth state of the microorganism, and the strains that can utilise or degrade the toxin are screened and isolated. Through further study, targeted degrading enzymes are obtained, and this strategy has achieved many successes (Cheng et al., 1969; Dai et al., 2015; Liu et al., 2016).

The gossypol, as an antinutritional factor (ANF) or natural plant toxin, has antifertility effects on humans and animals (Randel et al., 1992). The gossypol exists in cottonseed oil and meal (CM), and is a typical food or feed contaminant ( EFSA Panel on Contaminants in the Food 
Chain (CONTAM) et al.,2017). Through the classic screening method many researchers have discovered that some microorganisms, such as Aspergillus niger (AN) (Yang et al., 2012), Candida tropicalis (Mageshwaran et al., 2018), Aspergillus flavus (Yang et al., 2010) and Bacillus subtilis (Zhang et al., 2018), can degrade gossypol, and AN is the most reported microorganism. On the basis of previous screening strategies (Fig.1 B), AN that can grow in an agar solid medium with gossypol as the sole carbon source is isolated from cattle rumen liquid. The transcriptome of $\mathrm{AN}$ is obtained, and relevant genes or degrading enzymes involved in the degradation of gossypol in AN are attempted to be discovered. However, results have not met our expectations. Therefore, the rigor of the traditional screening strategy for toxin-degrading strains is questioned (Fig.1 C).

This study has confirmed the deficiencies of previous screening strategies for gossypol-degrading microorganisms (Fig.1 C) and expects to design a scientific and rigorous screening method for toxin-degrading microorganisms on the basis of existing methods. 

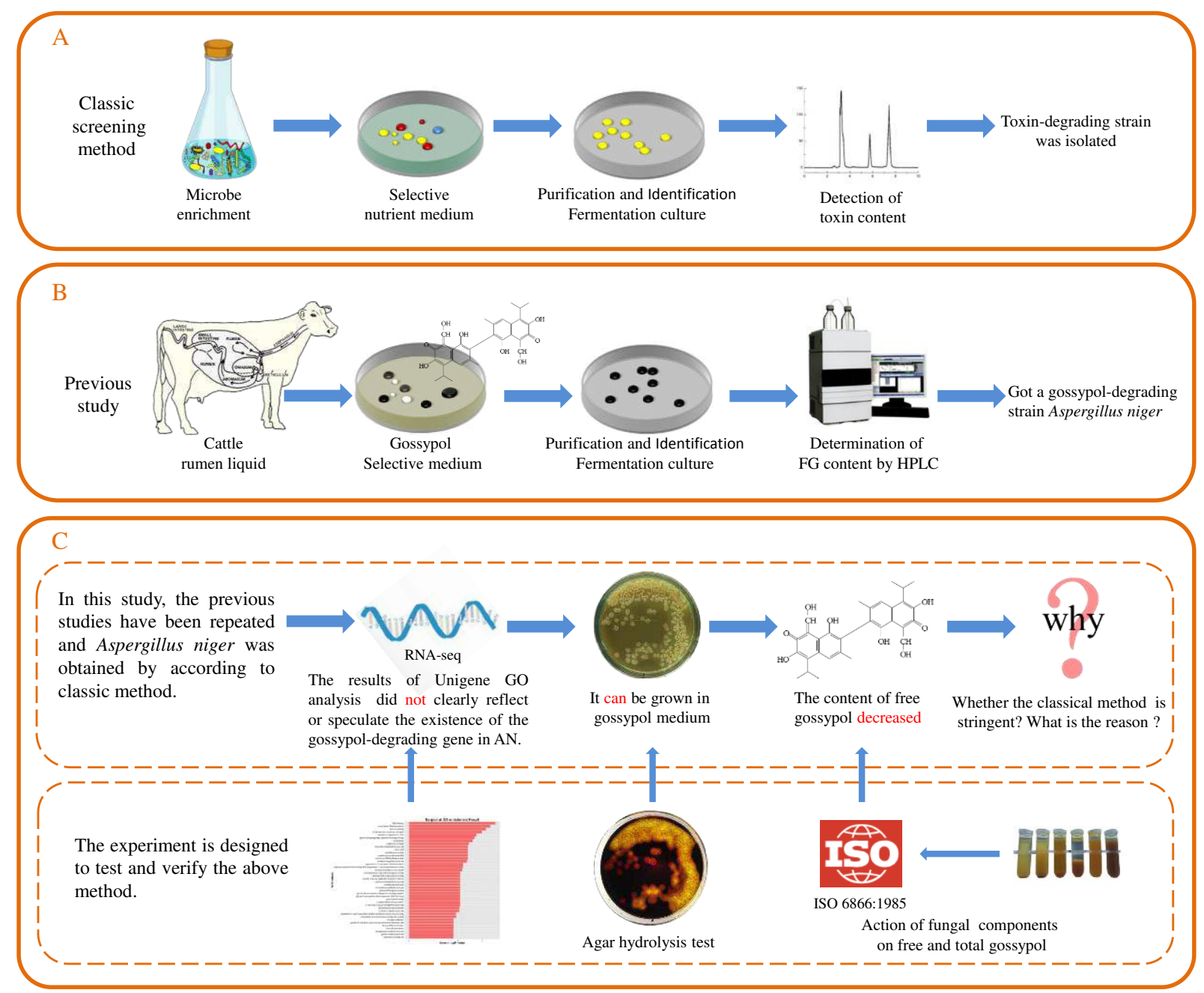

Fig. 1 Validation of the effectiveness of the classic toxin-degrading strain screening

\section{strategy}

A: Classic screening method; B: Previous study; C: The starting point of the study and the design of verification.

\section{Materials and Methods}

\section{Microbial strain}

Aspergillus niger which can grow in an agar solid medium with gossypol as the sole carbon source is isolated from cattle rumen liquid was obtained by classic screening strategy (Yang et al., 2012; Zhang et al., 2018; Lewis et al., 2020). The cattle rumen liquid samples were 
collected from Huangshan Junfeng ecological cattle farm.

\section{Transcriptome sequencing (RNA-seq) and the trait identification of AN}

AN cultured in potato dextrose agar (PDA, Hope Bio-technology Co., Ltd. Qingdao) was used as the control group, and AN cultured in PDA with 2\% acetate gossypol (AG, Ci Yuan Bio-technology Co., Ltd. Shaanxi) was used as the experimental group. The Illumina Hiseq ${ }^{\mathrm{TM}}$ 2500 high-throughput sequencing technology was used to sequence the transcriptome of AN under normal and 2\% AG conditions. Differentially expressed genes (DEG) were screened, and $2 \%$ AG response-related genes were analysed using the gene ontology (GO) and the KEGG (Kyoto Encyclopedia of Genes and Genomes) databases for annotation in AN, measured by Shanghai Biotechnology Co., Ltd.

The AN and spores were observed using scanning (SEM) and transmission (TEM) electron microscopy (Tizro et al., 2019).

\section{Growth in different media}

Solid medium were prepared as shown in Table 1, and an equal amount of the AN spore solution was inoculated and cultured at $30{ }^{\circ} \mathrm{C}$ for $24 \mathrm{~h}$. The growth of $\mathrm{AN}$ was observed and recorded. And after growing AN for $30 \mathrm{~h}$, the media were stained with Lugol's iodine solution (Fu et al., 2010) in the dark for 1 min, washed thrice with clean water and observed under a normal light source.

Table 1. Preparation of solid media for AN

\begin{tabular}{ll}
\hline No. & Medium components \\
\hline 1 & Sugar-free CzA $+0.5 \%$ AG \\
2 & Sugar-free CzA \\
3 & CzA
\end{tabular}


CzA: Czapek-Dox Medium $\left(\mathrm{NaNO}_{3} 2 \mathrm{~g}, \mathrm{~K}_{2} \mathrm{HPO}_{4} \mathrm{~g}, \mathrm{KCl} 0.5 \mathrm{~g}, \mathrm{MgSO}_{4}\right.$ 0.5g, $\mathrm{FeSO}_{4} 0.01 \mathrm{~g}$, Sucrose 30g, Agar 20g, distilled $\mathrm{H}_{2} \mathrm{O} 1000 \mathrm{~mL}$, natural $\mathrm{pH}, 121^{\circ} \mathrm{C}, 20 \mathrm{~min}$ ); Sugar-free $\mathrm{CzA}$ :

Czapek-Dox Medium without Sucrose.

\section{Preparation of the crude enzyme and the cell wall pellet suspension}

AN was inoculated in potato dextrose broth, and the crude enzyme and the cell wall suspension were prepared as follows.

(1) After $24 \mathrm{~h}$, the AN fermentation broth was collected (before spore production), subjected to repeated freeze-thaw and crushed using ultrasonic wave (in ice water) until no intact cell structure was found using a microscope (Zhang et al., 2021).

(2) The fermentation broth was centrifuged at $4{ }^{\circ} \mathrm{C}$ and $8000 \mathrm{rpm}$ for $10 \mathrm{~min}$. The supernatant and the fungus precipitate were collected. After washing the fungus precipitate thrice with aseptic water, the precipitate was resuspended to the original volume, and the cell wall pellet suspension was obtained.

(3) The supernatant was filtered using the millipore filter $(0.45 \mu \mathrm{m})$ (Sultana et al., 2018), and the crude enzyme was obtained and stored at $-20{ }^{\circ} \mathrm{C}$ for later use.

\section{Removal experiment of AG by AN components}

The experiment was designed and carried out in accordance with Table 2. Experimental groups were restored to normal temperature, and AG was added to make the concentration reach $0.5 \%$ and shaken well. Samples $1,2,3$ and 4 were used as experimental groups, and sample 5 was used as the blank control group. Samples were collected before and after standing at $30{ }^{\circ} \mathrm{C}$ in the dark for $24 \mathrm{~h}$ and stored at $-20{ }^{\circ} \mathrm{C}$ for testing. Before testing samples 

were calculated according to the formula Eq. (1).

Eq. (1): $\mathrm{Rr}=\frac{\mathrm{C} 1-\mathrm{C} 2}{\mathrm{C} 1} \times 100 \%$

Where, Rr: Removal rate, C1: Initial content of FG (or TG) and C2: Rest of FG (or TG)

Table 2. Experimental design of the gossypol removal

\begin{tabular}{ll}
\hline No. & Treatment methods \\
\hline 1 & Crude enzyme \\
2 & Crude enzyme inactivated using boiling water bath for 10 min \\
3 & Cell wall pellet suspension \\
4 & Cell wall pellet suspension inactivated using boiling water bath for 10 min \\
5 & Aseptic pure water \\
\hline
\end{tabular}

\section{Statistical analysis}

113 All statistical analyses were performed using the SPSS version 19 (IBM, Armonk, NY)

114 Statistical differences were determined using the Duncan's multiple range test, and

115 significance was defined as $P<0.05$.

116 Results

117 GO enrichment analysis of differentially expressed genes 


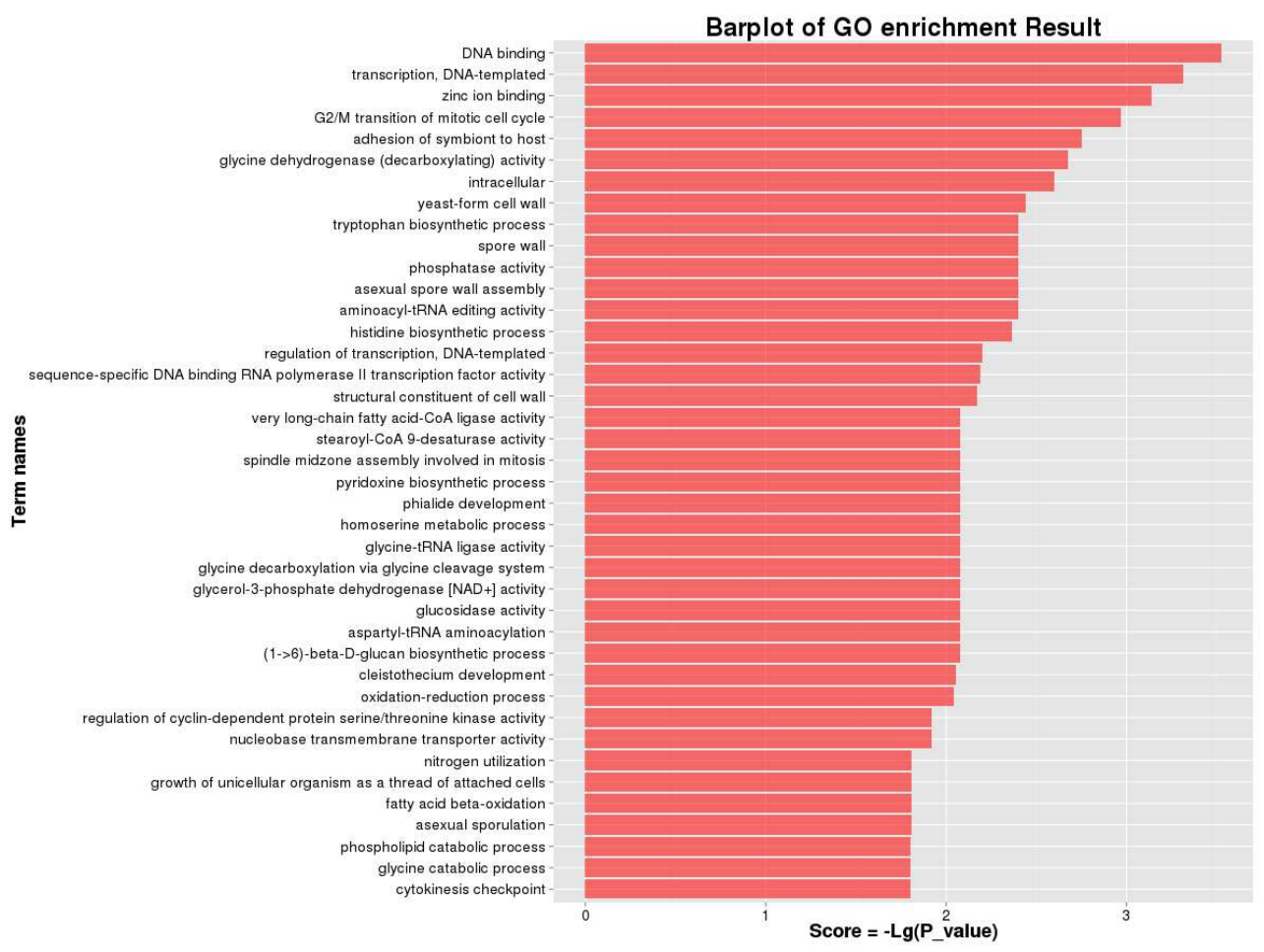

Fig. 2 GO functional annotation of differentially expressed genes under the gossypol stress

The Unigene GO analysis (Fig. 2) showed that 25112 results were annotated and divided into three functional groups, namely, biological process $(33.52 \%)$, molecular function $(28.65 \%)$ and cell component (37.83\%). The 10 most significant differences were DNA binding, transcription DNA-templated, zinc ion binding, G2/M transition of mitotic cell cycle, adhesion of symbiont to host, tryptophan biosynthetic process, asexual spore wall assembly, repair, adhesion and cell structure (Zámborszky, 2013; Swirnoff et al., 1995; Sharma et al., 2020). Results did not clearly reflect or speculate the existence of the gossypol-degrading gene in AN.

\section{Growth status in different media}


AN could grow on all media. At the same culture time, the degrees of growth and maturity followed the order: $\mathrm{CzA}>$ sugar-free $\mathrm{CzA}>$ sugar-free $\mathrm{CzA}+0.5 \% \mathrm{AG}>$ agar, and spores were produced except in agar (Fig. 3A). The AN in the agar medium was the weakest (Fig. 3 A4) followed by sugar-free CzA added with $0.5 \%$ AG (Fig. 3 A1). Results showed that screening AN by using agar medium added with $\mathrm{AG}$ as the sole carbon source was not rigorous.

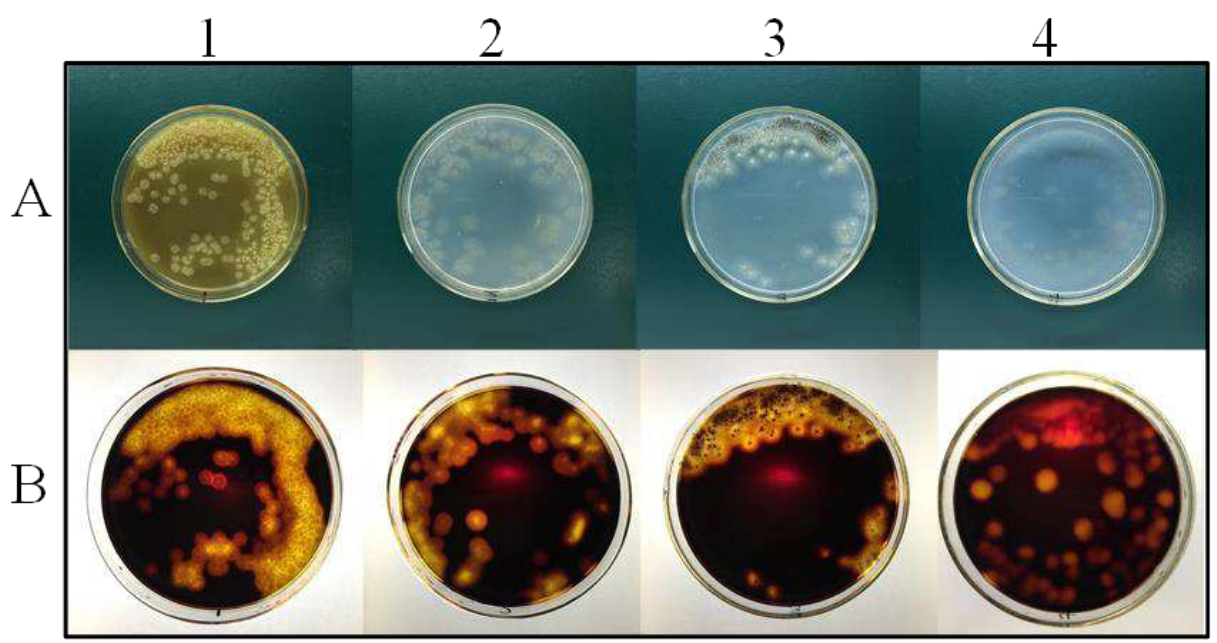

Fig. 3 Growth status of AN in media and results of Lugol's iodine staining

A: Before and B: after staining with Lugol's iodine

1: Sugar-free CzA + 0.5\% AG, 2: Sugar-free CzA, 3: CzA, 4: agar medium

\section{Lugol's iodine staining}

Four media were stained with Lugol's iodine after growing AN for $30 \mathrm{~h}$. Results are shown in Fig. 3B. After staining, corresponding bright spots were observed on the growth position of AN, and the surface of media were slightly dented. The result showed AN could secrete agarase to hydrolyse agar and utilise agar as carbon and energy sources.

\section{Morphological changes in AN}


A

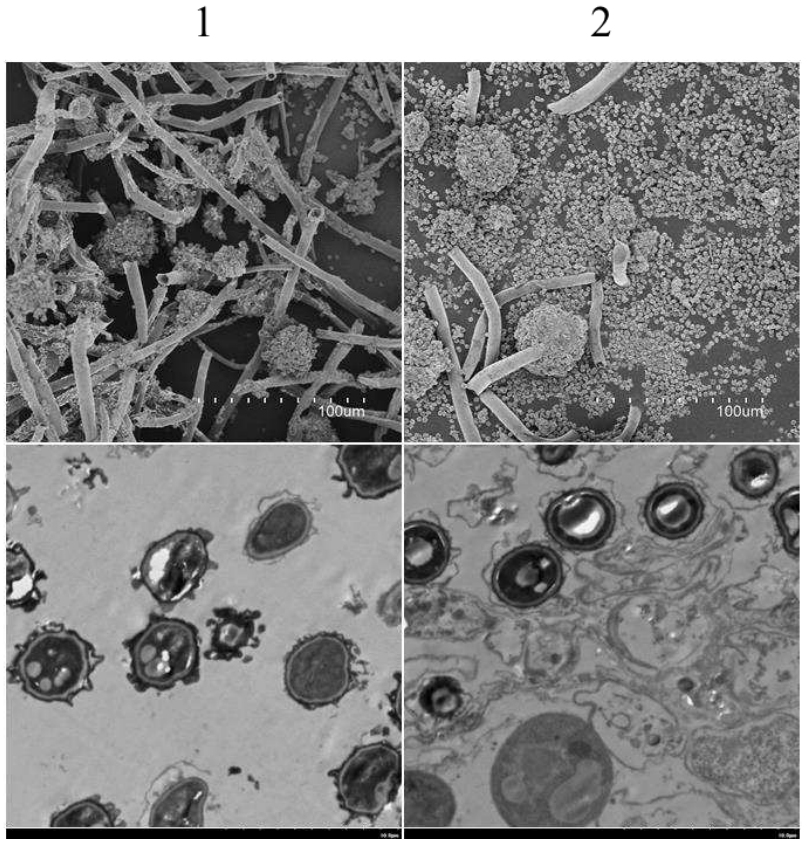

Fig. 4 Micrographs of $\mathrm{AN}$ and spores

149 A: SEM image of AN, B: TEM image of spores;

150 1: Growth of AN on PDA added with 2\% AG, 2: Growth of AN on PDA.

151 Under the gossypol stress, AN had increased hyphae and decreased number of spores

152 compared with AN grown on PDA alone (Fig. 4A). The spores grown on AG were deformed;

153 had blisters on the surface; thin, uneven and damaged cell wall and small ratio of organelles to

154 cell volume (Fig. 4B). Results showed that gossypol had a negative effect on the AN growth

155 and could cause a bubblelike structure to form on the spores' surface and structural injury in

156 the cell wall. However, AN had a certain tolerance to gossypol injury.

\section{Removal efficiency of fungal components on AG}




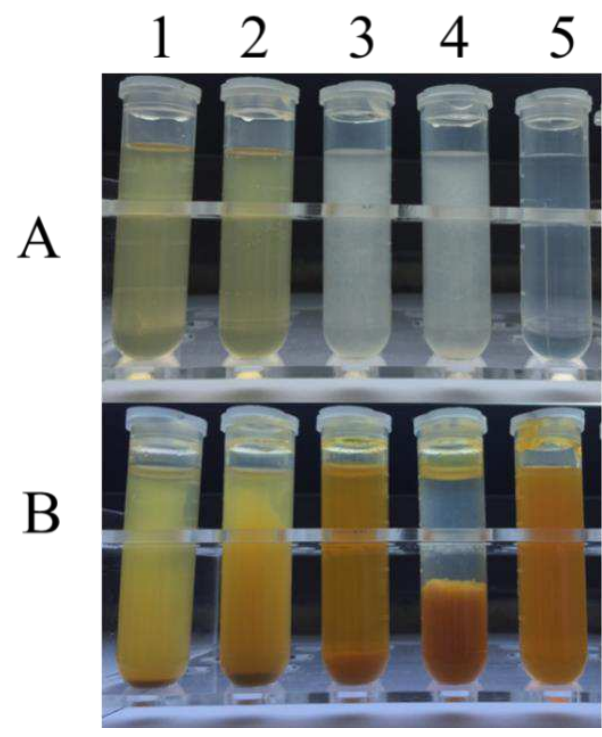

159 Fig. 5 Flocculation and sedimentation of fungal components in AG

160 A: Before and B: after adding AG;

161 1: Crude enzyme, 2: Inactivated crude enzyme, 3: Cell wall pellet suspension,

162 4: Inactivated cell wall pellet suspension, 5: Blank control (aseptic pure water).

163 AG was added to each group, and the mixture was placed at room temperature for $1 \mathrm{~h}$ (Fig. 5).

164 Flocculation and sedimentation were observed in all experimental groups. The influence of 165 the inactivated cell wall pellet (Fig. 5 B4) was most notable followed by that of the 166 un-inactivated cell wall pellet (Fig. 5 B3). 


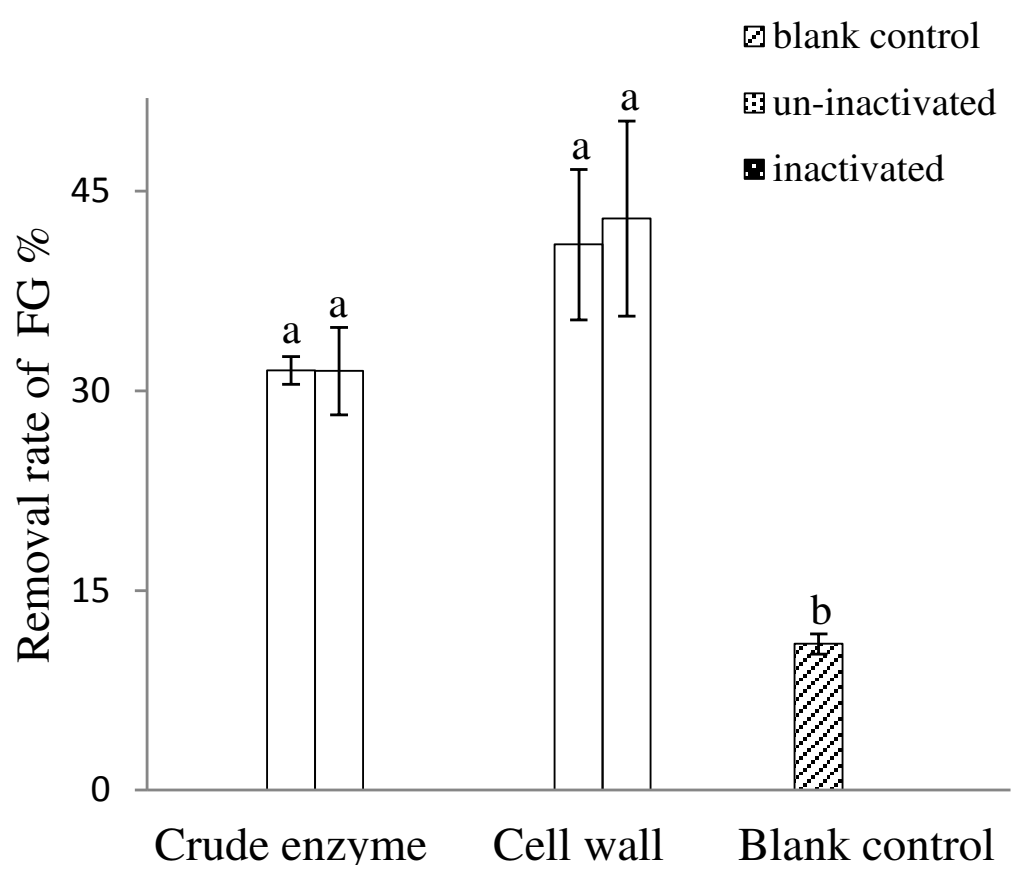

Fig. 6 Removal efficiency on FG by fungal components

Different lowercase letters indicate significant differences amongst treatment groups $(P<$

171 Compared with the control group, the crude enzyme and the cell wall of AN had significant

172 removal effects on the FG in the sample $(P<0.05$, Fig. 6$)$, and the inactivated cell wall group

173 had the highest removal rate (42.94\%). The FG removal rate of each component after 174 inactivation was higher than that before inactivation. The FG removal rates of cell wall groups 175 were higher than that of the crude enzyme groups, but no significant difference was observed 176 amongst experimental groups $(P>0.05)$. 


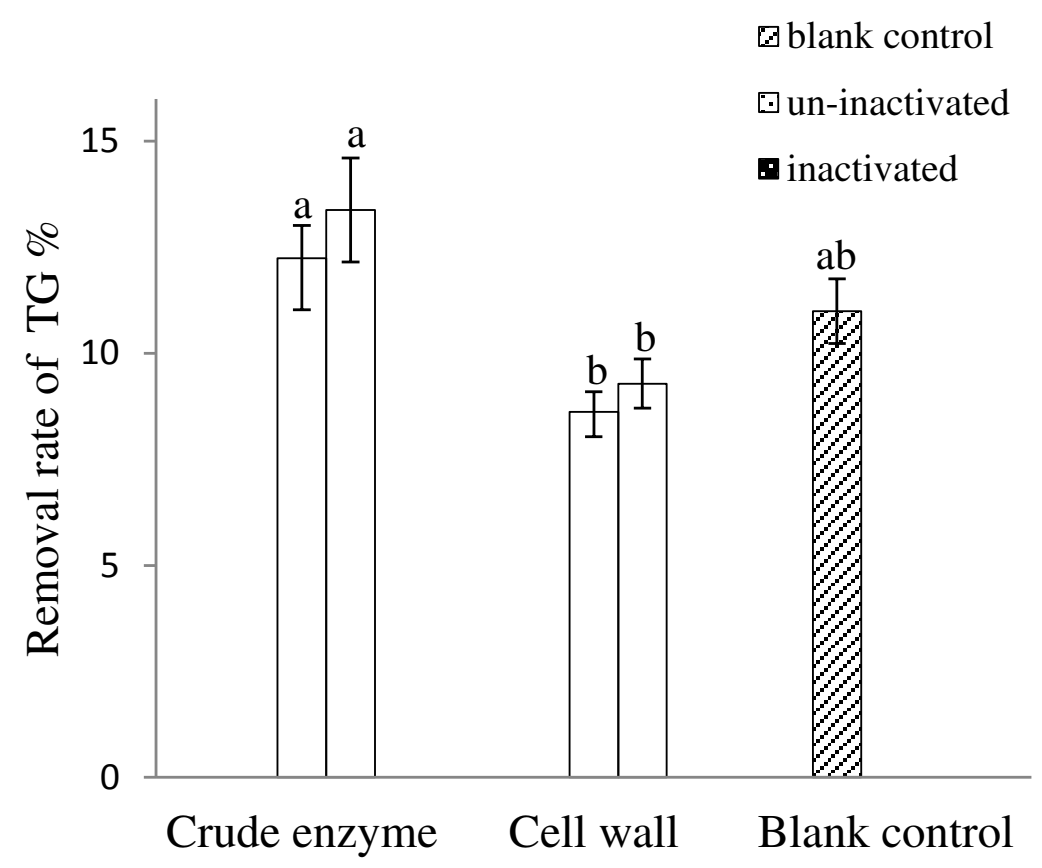

Fig. 7 Removal efficiency on TG by fungal components

Different lowercase letters indicate significant differences amongst treatment groups $(P<$

The components of AN had different removal effects on TG in the sample (Fig. 7). The removal rate of each group was low (all $<15 \%$ ), and the inactivated crude enzyme group had the highest TG removal rate $(13.38 \%)$. The FG removal rate of each component was higher after inactivation, but no significant difference was observed amongst the same component groups $(P>0.05)$. The crude enzyme group had significant differences amongst cell wall groups $(P<0.05)$, but no significant difference was observed between the control and the experimental groups $(P>0.05)$.

\section{Discussion}

In this experiment, AN can grow in four kinds of media, and screening the degrading strains simply by using an agar medium added with toxin as the sole carbon source is not rigorous. 
191

192

193

194

195

196

197

198

199

200

201

202

203

204

205

206

207

The Lugol's iodine solution can stain polysaccharides in agar into a dark brown colour and cannot stain the degraded oligosaccharides of agar (Fu et al., 2010). This solution has been used to visualise the agarase activity to screen the agarase production by microorganisms on a culture plate (Kawai, 2003). Similar to the AN in this study, some strains (Fu et al., 2010; Chi et al., 2014) can utilise agar to grow normally on the medium without any sugar, interfere with the expected experimental results and affect the experimenter to make a true judgment on the results.

Enzymes are metabolites secreted by living organisms and can be divided into primary (constitutive enzymes) and secondary (inducible enzymes) metabolites. This study first discovers that AN can secrete a constitutive enzyme that hydrolyses agar and utilise agar as sole carbon source. Therefore, we suspect that the gossypol-degrading enzyme of AN may not be a primary metabolite and that the gossypol cannot be directly utilised. This enzyme may be an inducible enzyme and can be secreted only under the condition of the gossypol as an inducer. In this study, the gossypol has a negative effect on the normal growth of AN. However, AN has a certain tolerance to the gossypol injury and produces active spores. Thus, AN may produce some special inducing enzymes under the induction of the gossypol. Given that the premise of the basic physiological metabolism of the cell is guaranteed, when exogenous stimuli interfere with the normal metabolism of cells or cause cell damage, certain proteins (such as Quinone oxidoreductase, Cytochrome p450, Catalase) are secreted abundantly by the cells, which are under the exogenous stimulus stress against exogenous substances and protect cells (Mcclain et al., 1999; Gu et al., 2020). Therefore, when screening 
for a toxin-degrading strain by using a medium with the toxin as the sole carbon (or nitrogen) and energy sources, the function of inducing enzymes may be overlooked by usual screening methods of the predecessors.

For the removal effect of FG (or TG), no significant difference is observed before and after inactivation in crude enzyme groups (or cell wall groups). The TG removal rate of each group is lower than the FG removal rate (Fig. 6 and Fig. 7). For AN, the FG removal function and metabolic enzymes may not be closely related. Heating promotes proteolysis and increases the content of free amino acids (Jiang et al., 2013; Chang et al., 2014;). The crude enzyme solution is composed of proteins, and the cell wall suspension also contains some little cell wall proteins. After heat inactivation, the content of free amino acids in the solution increases. The Maillard reaction occurs and is strengthened between the carbonyl group from the gossypol and free amino acids under ISO detection conditions. As a result, the TG content decreases, and the TG removal rates of the crude enzyme and the cell wall groups increase when inactivated. Significant differences between the crude enzyme and the cell wall groups are observed due to different protein contents. The gossypol is unstable at room temperature, and a small amount of gossypol is degraded (Nomeir et al., 1982). No significant difference in the TG removal rate is observed between the control and the crude enzyme or the cell wall group $(P>0.05)$. Thus, we believe that the decrease in TG content is not due to AN. Hyphae secrete enzymes that convert the organic material from media into small molecules that can be taken up by the fungus to serve as nutrients (Vinck et al., 2005). Hyphae in filamentous fungi also serve as store house for nitrogen and phosphorus in the form of DNA, 
233

234

235

236

237

giving hyphal tips the capability of persistent extension and foraging in new areas (Maheshwari, 2005). The inadequate nutrition in sugar-free medium prompts AN to increase absorbable nutrients indirectly by increasing the number of hyphae. The toxic effect of the gossypol prompts AN to extend away from the gossypol damage by increasing the number and the length of hyphae. The agar medium lacks minerals and is not enough to support the growth of AN. AN relies on stored nutrients in spore growth and grows weakly. The main components of the AN cell wall are chitin and glucan and used as biosorbent for heavy metals (Filipovic-Kovacevic et al., 2000). The removal effect of AN on FG may be due to the biosorption of fungi. The gossypol can destroy the structure of $\mathrm{AN}$ and increase the gene expression of fungus cell stress resistance, proliferation, tissue repair, adhesion and cell structure. The reduction in gossypol is more likely to be consumed by damaging AN or protein.

When screening toxin-degrading microorganisms, we usually regard the toxin as the sole carbon source, in the preparation of agar solid medium tend to ignore the agar could be a potential carbon source, moreover, in culturing, is likely to ignore the carbon dioxide $\left(\mathrm{CO}_{2}\right)$ (Peng et al., 2010 Steffens et al.,2021) in the air even light (Shi et al., 2008) may be a carbon or energy for some microorganisms. Thus, factors, such as agar, $\mathrm{CO}_{2}$, illumination and inducible enzymes should be fully considered.

HPLC has previously been employed to detect gossypol content changes, and this method can only detect free gossypol, while total gossypol can only be effectively detected by ISO 6866:1985 (Animal feeding stuffs-Determination of free and total gossypol). The 

be considered fully, basing on the change in total toxin to determine whether the target strain adsorbs or degrades toxins.

Besides, substances or toxins can be directly, exactly and efficiently analysed quantitatively and qualitatively by using a mass analyzer and other equipment (Zhou et al., 2016; Yan et al., 2018). The toxin can be more intuitive judged whether or not to be degraded or form a new substance. Yet the toxin degradation products by microorganisms usually have many characteristics, such as low content and mixed with a variety of complex substances, the composition is complicated and difficult to extract and purify. In addition, the mass analyser has high requirements on operating ambient detection conditions and is not suitable for use at the initial phases of screening degrading strains.

During the processes, such as agricultural production, the exploitation and the utilisation of resources, sewage treatment and food storage and processing. Various degrees of toxin pollution, including organochlorine pesticides, polycyclic aromatic hydrocarbons (PAHs), dyes, mycotoxins and others, are available, and most of them are refractory organic pollutants, bioaccumulative, persistent and toxic with carcinogenic and mutagenic activities (Jones et al., 1999; Kelly et al., 2007; Huen et al., 2014). The successful screening and the domestication of microorganisms with degradation effect on a certain pollutant are important for environmental 
protection and human health. When screening toxin-degrading strains by using a toxin as the sole external carbon source and the solid medium as gel, the selection strategy should be optimised, and control groups with agar, $\mathrm{CO}_{2}$ and light should be established at the same time. The screening scope can be extended to toxin-resistant organisms, and the content can be reduced by the inducible enzyme or consumed by the injury of resistant organism. The screening of pollutant-degrading strains is a tedious task and contingent in nature. Therefore, screening strategies should be fully considered when conducting strain screening for improved accuracy.

\section{Authors' contributions}

C Z, G S, H W, D L, Y X, Y Z, M C, L C designed and analyzed the experiments. C Z and G S performed the experiments. $\mathrm{C} \mathrm{Z}$ and $\mathrm{L} \mathrm{C}$ interpreted the data and wrote the manuscript, and were major contributors in writing the manuscript. All authors read and approved the final manuscript.

\section{Acknowledgements}

This work was supported by grants from the Science and Technology Research Projects of Anhui Province [201904b11020043] and the National Natural Science Foundation of China [31872418].

\section{Competing interests}

The authors declare that they have no competing interests.

\section{References}

Chang, Y.N., Zhao, G.M., Liu, Y.X., Li, M.Y., Huang, X.Q., Sun, L.X., 2014. Changes of free 
amino acids in chicken and its broth during cooking. Sci Technol Food Ind. 35,

298

299

300

301

302

303

304

305

306

307

308

309

310

311

312

313

314

315

Cheng, K.J., Jones, G.A., Simpson, F.J., Bryant, M.P., 1969. Isolation and identification of rumen bacteria capable of anaerobic rutin degradation. Can J Microbiol. 15, 1365-1371. doi: 10.1139/m69-247

Chi, W.J., Park, D.Y., Seo, Y.B., Chang, Y.K., Lee, S.Y., Hong, S.K., 2014. Cloning, expression and biochemical characterization of a novel GH16 $\beta$-agarase AgaG1 from Alteromonas sp. GNUM-1. Appl Microbiol Biot. 98, 4545-4555. doi: $10.1007 / \mathrm{s} 00253-014-5510-4$

Dai, P.F., Cai, T.M., Wang, L., Zhang, W.M., Lu, L.V., Shao, Y., Zhao, X.M., Qian, D.U., Yang, J., Li, L.F., Shi, J.J., 2015. Isolation, identification, degradation characteristics and pathway of a nicosulfuron-degrading bacteria. Environ Sci Technol. 28, 12-21. doi:

\subsection{9/j.issn.1674-4829.2015.04.003}

EFSA Panel on Contaminants in the Food Chain (CONTAM)., Knutsen, H.K., Barregård, L., Bignami, M., Brüschweiler, B., Ceccatelli, S., Dinovi, M., Edler, L., Bettina, G.K., Hogstrand, C., Hoogenboom, L.(Ron)., Nebbia, C.S., Oswald, I.P, Petersen, A., Rose, M., Roudot, A.C., Schwerdtle, T., Vleminckx, C., Vollmer, G., Wallace, H., Alexander, J., Cottrill, B., Mackay, K., 2017. Presence of free gossypol in whole cottonseed. EFSA J. 15, e04850. doi: 10.2903/j.efsa.2017.4850

Filipovic-Kovacevic, Ž., Sipos, L., Briški, F., 2000. Biosorption of Chromium, copper, nickel and zinc ions onto fungal pellets of Aspergillus niger 405 from aqueous solutions. Food 
Fu, X.T., Kim, S.M., 2010. Agarase: Review of major sources, categories, purification method. enzyme characteristics and applications. Mar Drugs. 8, 200-218. doi:

\section{$10.3390 / \mathrm{md} 8010200$}

Ghazali, F.M., Rahman, R.N.Z.A., Salleh, A.B., Basri M., 2004. Biodegradation of hydrocarbons in soil by microbial consortium. Int Biodeter Biodegr. 54, 61-67. doi:

\subsection{6/j.ibiod.2004.02.002}

Gu, C., Hou, C., Zhang, S., 2020. miR-425-5p improves inflammation and septic liver damage through negatively regulating the RIP1-mediated necroptosis. Agents and Actions. 69, 1-10. doi: 10.1007/s00011-020-01321-5

Hazrat, A., 2010. Biodegradation of Synthetic Dyes-A Review. Water Air Soil Poll. 213, 251-273. doi:10.1007/s11270-010-0382-4

Huen, K., Yousefi, P., Bradman, A., Yan. L., Harley, K.G., Kogut, K., Eskenazi, B., Holland N., 2014. Effects of age, sex, and persistent organic pollutants on DNA methylation in children. Environ Mol Mutagen. 55, 209-222. doi: 10.1002/em.21845

Jiang, M., Dong, M.S., Rui, X., Li, W., Chen, X.H., 2013. Effects of high pressure homogenization and heat treatment on protein solubility in soymilk. Food Sci. 34, 125-130. doi: 10.7506/spkx1002-6630-201321026

Ji, C., Zhao, L.H., 2010. Research and prospects of aflatoxin biological detoxification. Chinese Journal of Animal Nutrition. 22, 241-245. doi: 
Jones, K.C., Voogt, P.D., 1999. Persistent organic pollutants (POPs): state of the science. Environ Pollut. 100, 209-221. doi: 10.1016/s0269-7491(99)00098-6

Kawai, S.K., 2003. Purification and characterization of an extracellular $\beta$-agarase from Bacillus sp. MK03. J Biosci and Bioeng. 95, 456-463. doi: 10.1016/S1389-1723(03)80063-4

Kelly, B.C., Konomou, M.G., Blair, J.D., 2007. Food Web-Specific biomagnification of persistent organic pollutants. Science. 317, 236-239. doi: 10.1126/science.1138275

Lewis, W.H., Tahon, G., Geesink, P., Sousa, D.Z., Ettema, T.J.G., 2020. Innovations to culturing the uncultured microbial majority. Nat Rev Microbiol. doi: $10.1038 / \mathrm{s} 41579-020-00458-8$

Liu, B., Ju, M., Liu, J., Wu, W., Li, X., 2016. Isolation, identification, and crude oil degradation characteristics of a high-temperature, hydrocarbon-degrading strain. Mar Pollut Bull. 106, 301-307. doi: 10.1016/j.marpolbul.2015.09.053

Mageshwaran, V., Sharma, V., Chinnkar, M., Parvez, N., Krishnan, V., 2018. Biodegradation of gossypol by mixed fungal cultures in minimal medium 1. Appl Biochem and Micro+. 54, 301-308. doi: 10.1134/S0003683818030092

Maheshwari, M., 2005. Nuclear behavior in fungal hyphae. Fems Microbiol Lett. 249, 7-14. doi: $10.1016 /$ j.femsle.2005.06.031

Mcclain, C.J., Barve, S., Deaciuc, I., Kugelmas, M., Hill, D., 1999. Cytokines in Alcoholic Liver Disease. Sem Liver Dis. 19, 205-219. doi:10.1055/s-2007-1007110

Nomeir, A.A., Abou-Donia, M.B., 1982. Gossypol: High-performance liquid chromatographic 
analysis and stability in various solvents. J Am Oil Chem Soc. 59, 546-549. doi:

\subsection{7/BF02636320}

Peng, Y.L, Zhao, H.Z., Yang, Q.Z., Song, J., 2010. Progress in microbial and enzyme immobilization of carbon dioxide. Chemistry and Bioengineering. 27, 10-13. doi: 10.3969/j.issn.1672-5425.2010.07.003

Randel, R.D., Chase, C.C., Wyse, S.J., 1992. Effects of gossypol and cottonseed products on reproduction of mammals. J Anim Sci. 70, 1628-1638. doi: $10.1080 / 00071669208417483$

Sharma. D., Singh, A., Pathak, M., Kaur, L., Kumar, V., Roy, B.G., Ojha, H., 2020. DNA binding and antiradical potential of ethyl pyruvate: Key to the DNA radioprotection. Chem-biol Interact. 332, 1-9. doi: 10.1016/j.cbi.2020.109313

Shi, Y.Z., Zhang, Q.G., Wang, Y., Jing, Y.Y., 2008. Experimental study on continuous culture of photosynthetic bacteria for hydrogen production from biomass. Transactions of the CSAE. 24, 218-221. doi: 10.3321/j.issn:1002-6819.2008.06.046

Steffens, L., Pettinato, E., Steiner, T.M, Mall, A., König, S., Eisenreich, W., $2021 \mathrm{High} \mathrm{CO}_{2}$ levels drive the TCA cycle backwards towards autotrophy. Nature. 592, 784-788. doi: $10.1038 / \mathrm{s} 41586-021-03456-9$

Sultana, N., Saha, R., 2018. Studies on potential application of crude keratinase enzyme from Stenotrophomonas sp. for dehairing in leather processing industry. J Environ Biol. 39, 324-330. doi: 10.22438/jeb/39/3/MRN-572

Swirnoff, A.H., Milbrandt J., 1995. DNA-binding specificity of NGFI-A and related zinc 
finger transcription factors. Mol and Cell Biol. 15, 2275-2287. doi:

Tizro, P., Choi, C., Khanlou, N., 2019. Sample Preparation for Transmission Electron 10.1093/brain/awh544 Microscopy. Methods Mol Biol. 1897, 417-424. doi: 10.1007/978-1-4939-8935-5_33

Vinck, A., Terlou, M., Pestman, W.R., Martens, E.P., Ram, A.F., van den Hondel, C.A.M.J.J., Wösten, H.A.B., 2005. Hyphal differentiation in the exploring mycelium of Aspergillus niger. Mol Microbiol. 58, 693-699. doi: 10.1111/j.1365-2958.2005.04869.x

Yang, X., Weng, X.Y., Guo, J.L., Sun, J.Y., 2010. Screening and Identification of Gossypol-degraded Strains Isolated from a soil microcosm. Cotton Sci. 22, 539-546. doi: $10.1080 / 00949651003724790$

Yang, X., Sun, J.Y., Guo, J.L., Weng, X.Y., 2012. Identification and proteomic analysis of a novel gossypol-degrading fungal strain. J Sci Food Agr. 92, 943-951. doi: $10.1002 /$ jsfa.4675

Yan, J., Feng, S., Wu, T., Liu, R.H., Tsao, R., 2018, Chemical constituents and antioxidant activity of flaxseed during germination. Journal of Chinese Mass Spectrometry Society. 39, 112-119. doi: 10.7538/zpxb.2017.0003

Zámborszky, J., 2013. Cell Cycle Transitions, G 2 /M. Encyclopedia of Systems Biology. 329-333. doi: 10.1007/978-1-4419-9863-7_38

Zhang, C., Yu, X., Xu, H.Q, Cui, G.Y, Chen, L.J, 2021. Action of Bacillus natto 16 on deoxynivalenol (DON) from wheat flour. J Appl Microbiol. doi: 10.1111/jam.15094

Zhang, Y., Zhang, Z., Dai, L., Liu, Y., Cheng, M., Chen, L., 2018. Isolation and 
